\title{
Surgical repair of tricuspid atresia
}

\author{
F. FONTAN and E. BAUDET \\ Centre de Cardiologie, Université de Bordeaux II, Hôpital du Tondu, Bordeaux, France
}

\begin{abstract}
Surgical repair of tricuspid atresia has been carried out in three patients ; two of these operations have been successful. A new surgical procedure has been used which transmits the whole vena caval blood to the lungs, while only oxygenated blood returns to the left heart. The right atrium is, in this way, 'ventriclized', to direct the inferior vena caval blood to the left lung, the right pulmonary artery receiving the superior vena caval blood through a cava-pulmonary anastomosis. This technique depends on the size of the pulmonary arteries, which must be large enough and at sufficiently low pressure to allow a cava-pulmonary anastomosis. The indications for this procedure apply only to children sufficiently well developed. Younger children or those whose pulmonary arteries are too small should be treated by palliative surgical procedures.
\end{abstract}

Only palliative operations (systemic vein to pulmonary artery anastomosis; systemic artery to pulmonary artery anastomosis) have been performed in tricuspid atresia. Although these procedures are valuable, they result in only a partial clinical improvement, because they do not suppress the mixture of venous and oxygenated blood.

We have initiated a corrective procedure for tricuspid atresia, which completely suppresses blood mixing. The entire vena caval return undergoes arterialization in the lungs and only oxygenated blood comes back to the left heart. This procedure is not an anatomical correction, which would require the creation of a right ventricle, but a procedure of physiological pulmonary blood flow restoration, with suppression of right and
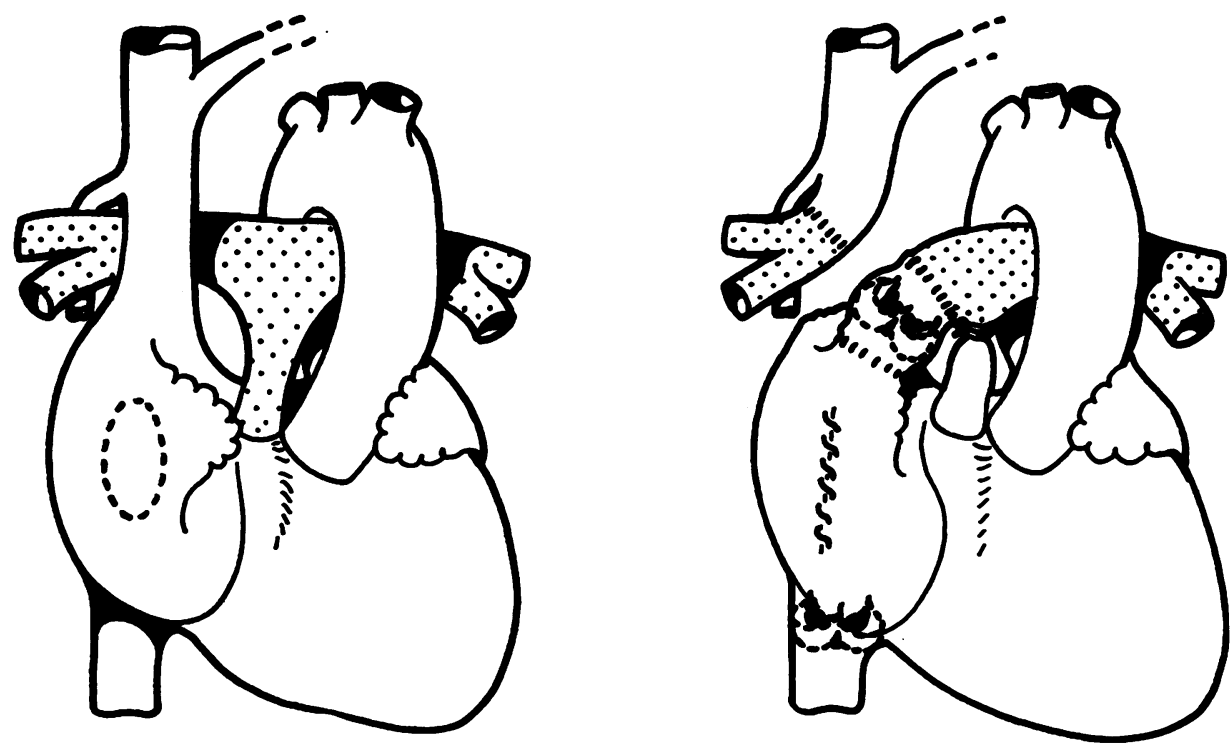

FIG. 1. Case 2. Tricuspid atresia type II B. Drawing illustrates steps in surgical repair: (1) end-to-side anastomosis of distal end of right pulmonary artery to superior vena cava; (2) end-to-end anastomosis of right atrial appendage to proximal end of right pulmonary artery by means of an aortic valve homograft; (3) closure of atrial septal defect; (4) insertion of a pulmonary valve homograft into inferior vena cava; and (5) ligation of main pulmonary artery. 
left blood mixing. This new surgical procedure has been used in three patients and has been successful in two of them; the first case has been followed satisfactorily for 30 months. The indications for this procedure apply only to children who are sufficiently well developed, without pulmonary arterial hypertension.

Palliative operations remain valuable in other patients and will permit many of them to have a secondary corrective procedure.

\section{SURGICAL TECHNIQUE}

The purpose of the operation is to drain the whole vena caval blood to the pulmonary arteries (Fig. 1): the superior vena cava is anastomosed to the distal end of the right pulmonary artery, according to Glenn's procedure; the proximal end of the right pulmonary artery is anastomosed to the right atrium ; so, after the atrial septal defect has been closed, the blood of the inferior vena cava is drained towards the left pulmonary artery. The main pulmonary artery is ligated at the point where it leaves the right hypoplastic ventricle, to prevent ventricular blood entering the left lung. In short, the right atrium is used to propel inferior vena caval blood through the left lung. To facilitate this function, the right atrium is provided with two aortic or pulmonary valve homografts: one is inserted into the inferior vena cava at its junction with the right atrium, to prevent blood reflux into the inferior vena cava during atrial systole ; the other is used as an anastomosis between the right atrial appendage and the proximal end of the right pulmonary artery, so that, during atrial diastole, there is no reflux from the left pulmonary artery into the right atrium.

The operation is performed through a median sternotomy. After the pericardium has been opened, the heart is examined to confirm the preoperative diagnosis of tricuspid atresia type. The pulmonary arteries also have to be examined carefully to ensure that their size is large enough to permit a cava-pulmonary anastomosis. In addition, it is necessary to measure the pressures in the pulmonary artery, thus making sure that there is no pulmonary arterial hypertension, which would be a contraindication to cavapulmonary anastomosis. This information, suspected from catheterization and angiocardiography, can only be corroborated during operation.

The surgical repair begins with the classic cavapulmonary anastomosis between the distal end of the right pulmonary artery and the right posterolateral aspect of the superior vena cava. End-to-side anastomosis is made, using a Blalock continuous suture (Fig. 2). But the superior vena cava is not yet transected at its entry into the right atrium, because it must be used for superior vena caval cannulation during cardiopulmonary bypass. This transection must be carried out as the last step of the operation.

The proximal end of the right pulmonary artery is then anastomosed to the right atrium by means of an

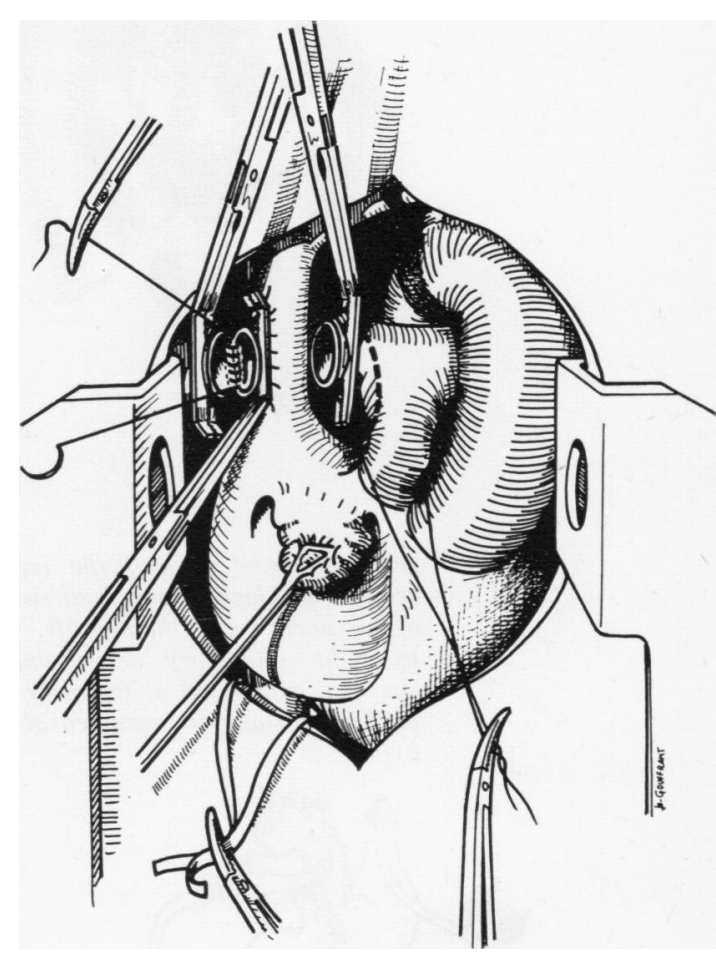

FIG. 2. First step of the repair: end-to-side anastomosis of distal end of right pulmonary artery to superior vena cava (Glenn, 1958). Drawing illustrates bevelling of proximal end of right pulmonary artery to ensure a correct fit with the aortic valve homograft (see Fig. 3).

aortic valve homograft (Fig. 3); the aortic wall is tailored to an adequate length; the origin of the right pulmonary artery can also be enlarged by bevelling up to the main pulmonary artery in order to achieve a good fit with the homograft (Fig. 2). End-to-end anastomosis is made using a continuous suture. The homograft (a short segment of the anterior mitral leaflet and septum below the aortic cusps has been kept) is end-to-end anastomosed to the right atrial appendage. There is no problem of fit with the atrial appendage which is, in tricuspid atresia, widely dilated, but fleshy tissues in the atrial appendage should be resected so that they do not hinder blood flow. Such a homograft was used in our second and third cases. In the first case, a younger child, we did not have a small enough homograft. We anastomosed the proximal end of the right pulmonary artery directly to the left lateral side of the upper part of the right atrium (Figs 7 and 8).

The operation then proceeds under cardiopulmonary bypass (Fig. 4), at flow rates of 2 to $2 \cdot 2$ litres/ $\mathrm{min} / \mathrm{m}^{2}$ at normothermia. The duration of cardiopulmonary bypass is about $\mathbf{4 0}$ minutes. The ascending 


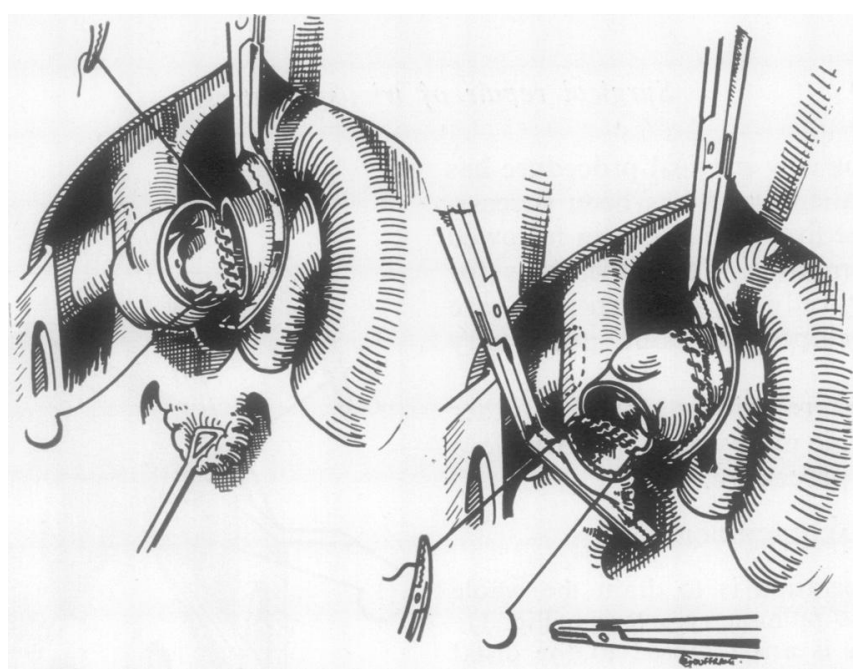

FIG. 3. Second step of the repair: end-to-end anastomosis of right atrial appendage to proximal end of right pulmonary artery by means of an aortic valve homograft. Drawing illustrates superior vena cava to right pulmonary artery anastomosis, but superior vena cava is not yet transected at its entry into right atrium because it must be used for superior vena caval cannulation during cardiopulmonary bypass.

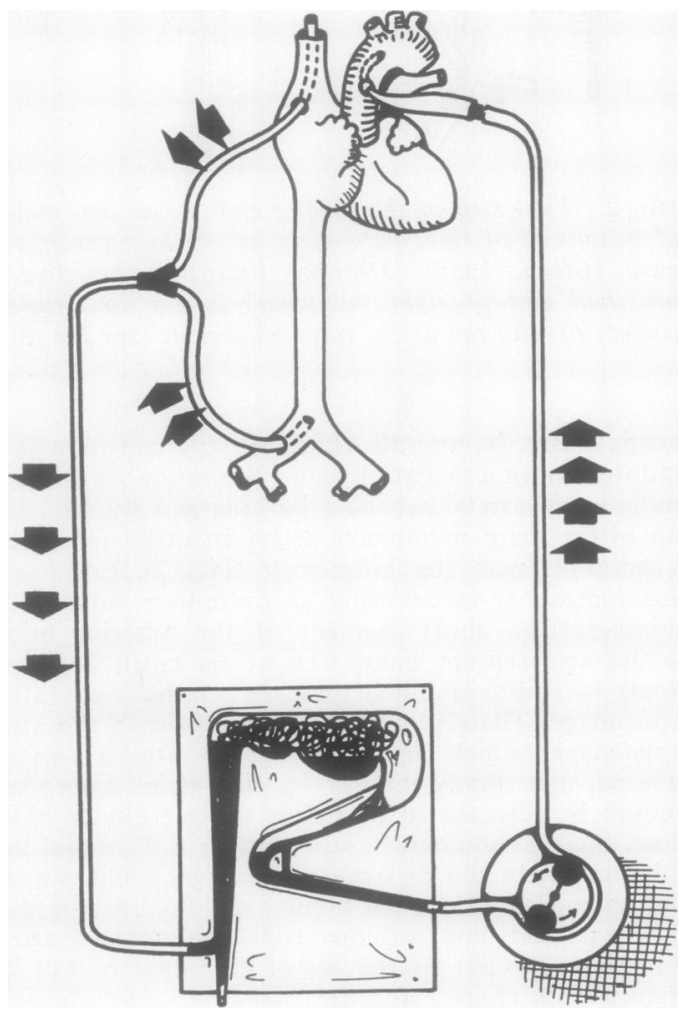

FIG. 4. Sketch of cardiopulmonary bypass, with cannulation of ascending aorta, superior vena cava, and right iliac vein. aorta is cannulated; the superior vena cava is cannulated through a purse-string suture slipped on between the right atrial appendage and the superior vena cava. The inferior vena cava is cannulated by means of the right external iliac vein so that the catheter does not prevent the insertion of the valve homograft into the inferior vena cava level with its junction with the right atrium. When the bypass is started, the superior vena cava is snared by an umbilical tape above the right cava-pulmonary anastomosis and the inferior vena cava is clamped just below its entry into the right atrium. The left ventricle is vented. The aorta is cross-clamped. After the right atrium has been opened (Fig. 5), the atrial septal defect is closed ; a pulmonary valve homograft is inserted into the inferior vena caval orifice. This homograft is prepared in the following manner: the whole subvalvular tissue is resected and only 2 to $3 \mathrm{~mm}$ of the arterial wall above the cusps is kept to suture the homograft to the atrial wall, using a continuous suture. There is no fear of harm to the bundle of His if the suture is passed sufficiently far behind the coronary sinus.

After the atriotomy has been closed, the air evacuated, and the clamps removed, the main pulmonary artery is ligated or transected. Cardiopulmonary bypass is discontinued as soon as cardiac action is vigorous. When the systemic pressure is above 100 $\mathrm{mmHg}$, the same or slightly higher pressures are looked for in the superior vena cava, the right atrium, and the pulmonary artery as were measured before bypass.

After the cannulae have been removed, the superior vena cava is transected between two clamps at its entry into the right atrium and both ends are sutured (Fig. 6). 


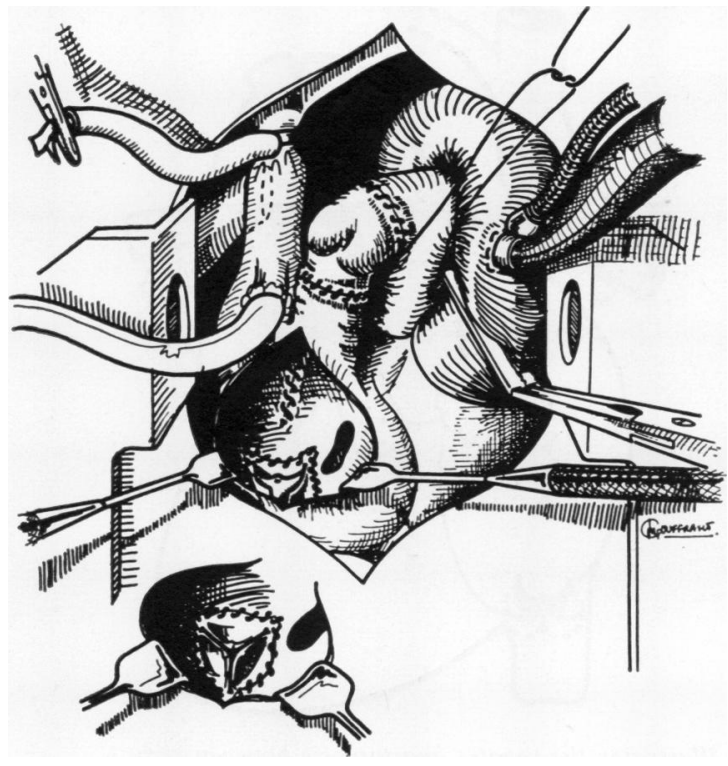

FIG. 5. Third step of the repair, under cardiopulmonary bypass: closure of atrial septal defect and insertion of a pulmonary valve homograft into inferior vena cava level with right atrium. Superior vena cava is cannulated through a purse-string suture, slipped on between right atrial appendage and superior vena cava, and it is snared by an umbilical tape above cava-pulmonary anastomosis.

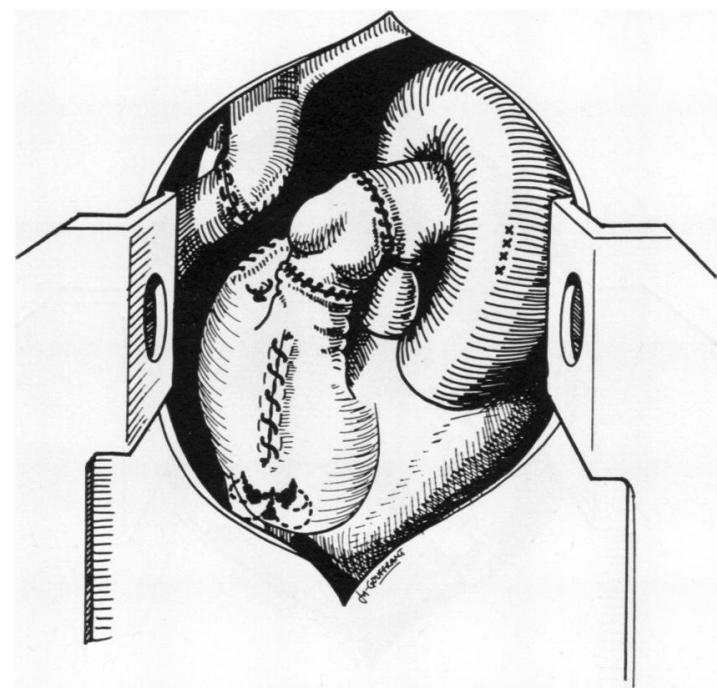

FIG. 6. The operation at completion, after the last step of the repair: superior vena cava is transected at its entry into right atrium and main pulmonary artery is ligated.
The pericardium is closed in the upper part without compromising the different anastomoses. The pericardial cavity and the anterior mediastinum are drained; the sternum is reapproximated with wire sutures and the subcutaneous tissue and skin are closed.

\section{CASE REPORTS}

CASE 1 Our first patient, C.F., underwent operation at the age of 12 years.

The face and extremities had been cyanosed from the age of 6 months and the cyanosis had gradually progressed; at the same time, exertional dyspnoea had appeared when she was admitted to hospital in 1961 , at the age of 6 years, for haemodynamic investigations.

Cyanosis was marked. There was clubbing of the fingers and toes. A loud systolic murmur was heard at the apex, radiating along the left of the sternum, with an accentuated second heart sound. Blood pressure was $100 / 60 \mathrm{mmHg}$. Manifestations of cardiac failure were not noted. A blood count showed $7,000,000 \mathrm{RBC} / \mathrm{mm}^{3}$.

Cardiac catheterization and angiocardiography revealed a type I B tricuspid atresia, with pulmonary arteries of good size. The possibility of a cava-pulmonary anastomosis was noted. The child left hospital and was lost sight of.

She was later readmitted to hospital in April 1968. She was very erythrocyanotic and manifested exertional symptoms-dyspnoea and frequent episodes of tachycardia. No signs of cardiac failure were noted. A blood count showed $7,800,000 \mathrm{RBC} / \mathrm{mm}^{3}$ and the haematocrit was $80 \%$.

Operation (Fig. 7) was performed on 25 April 1968 through a median sternotomy. The findings were tricuspid atresia without transposition of the great vessels (type I B) but with pulmonary arteries of good size and low intra-arterial pressure $(15.0 \mathrm{mmHg})$. A superior vena cava to pulmonary artery anastomosis and an anastomosis between the atrium and the proximal end of the right pulmonary artery were carried out (Fig 8); the azygos vein was not ligated. Then, under cardiopulmonary bypass, the atrial septal defect was closed, a pulmonary valve homograft was inserted into the inferior vena cava level with the right atrium, and the main pulmonary artery was ligated. After the cardiopulmonary bypass had been discontinued, the superior vena cava was divided level with the right atrium below the cava-pulmonary anastomosis (Fig. 9).

The initial postoperative course was very satisfactory: cyanosis disappeared. The patient was in sinus rhythm at 90 per minute and the blood pressure was $120 / 60 \mathrm{mmHg}$. Ventilation and haematosis were satisfactory. The venous pressure was not raised.

Twenty-four hours postoperatively anuria developed quite suddenly, but metabolic disorders were corrected by one haemodialysis only, while a moderate melaena appeared. The following day urinary 

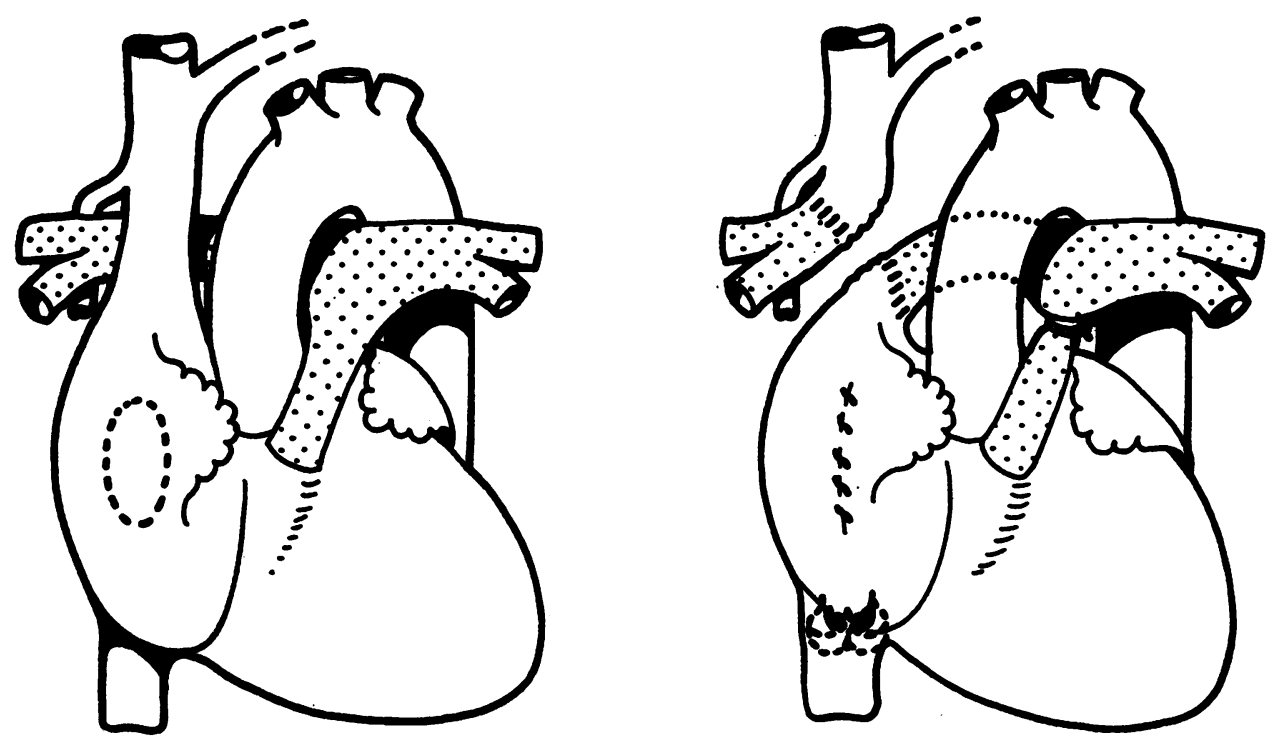

FIG. 7. Case 1. Tricuspid atresia type I B. Drawing illustrates the repair: anastomosis between right atrium and proximal end of right pulmonary artery was made without interposition of an aortic valve homograft.

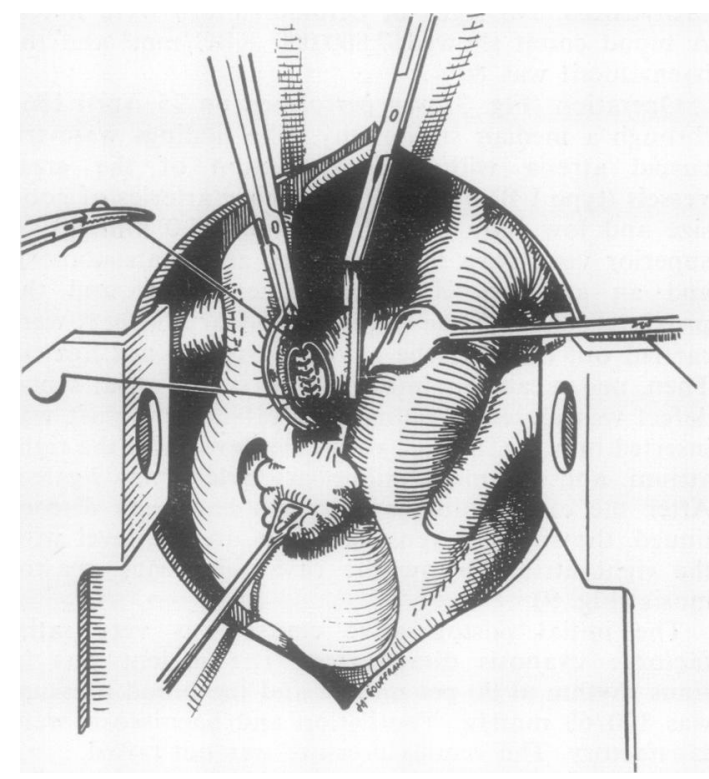

FIG. 8. End-to-side anastomosis of proximal end of right pulmonary artery to left lateral side of upper part of right atrium.

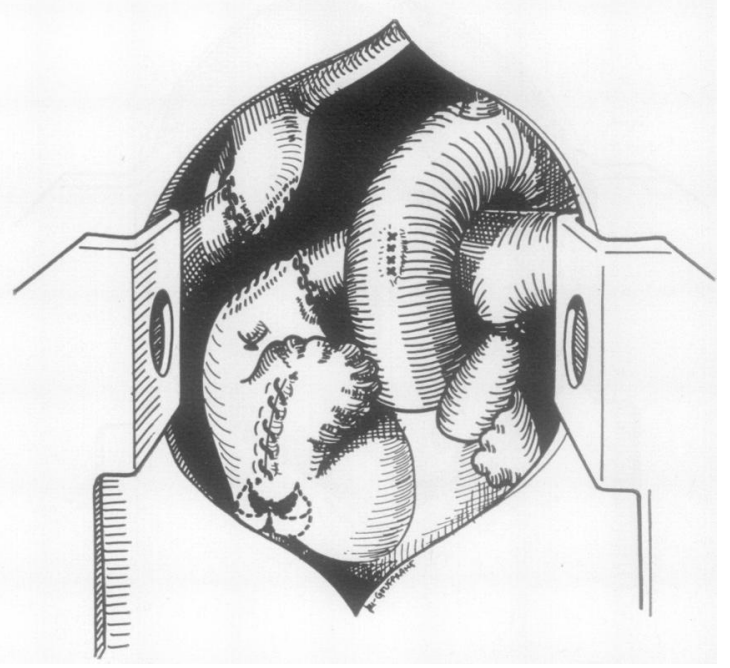

FIG. 9. Case 1. Appearance at completion of operation. 뭉 
function returned, after the patient's legs had been raised to improve the stagnant inferior vena caval circulation. From that moment there was no further urinary problem, but intravenous urography revealed a bilateral congenital hydronephrosis.

One month postoperatively a right serofibrinous pleural effusion required suction drainage for a few days.

The child was examined regularly after discharge from hospital and she is quite well after a 30 months' follow-up; she has grown normally and does not show exertional symptoms; she is no longer cyanotic and there is no oedema of the inferior limbs. The liver is just palpable below the costal edge. A chest radiograph shows normal pulmonary vascularity of both lungs. An electrocardiogram indicates a regression of the right atrial hypertrophy. A blood count showed $4,400,000 \mathrm{RBC} / \mathrm{mm}^{3}$ and the haematocrit was $52 \%$. Digitalis and diuretics in standard doses were prescribed. Anticoagulants were not used postoperatively. Physical examination is normal and there is no longer a systolic murmur.

The following postoperative haemodynamic and angiocardiographic investigations were made:

(1) The pressure curve in the superior vena cava fluctuated between 2.0 and $16.0 \mathrm{mmHg}$ with breathing movements. On the other hand, in the inferior vena cava and the right atrium, the pressure was stable, about $15.0 \mathrm{mmHg}$ (Table I).

\section{T ABLE I}

\begin{tabular}{|c|c|c|c|c|c|}
\hline & \multirow{2}{*}{$\begin{array}{c}\mathbf{R B C} \\
\text { per } \mathbf{m m}^{3}\end{array}$} & \multirow{2}{*}{$\underset{\%}{\text { Haematocrit }}$} & \multicolumn{3}{|c|}{ Pressure $(\mathrm{mmHg})$} \\
\hline & & & SVC & IVC & $\mathbf{R A}$ \\
\hline $\begin{array}{rr}\text { Preoperative } \begin{array}{r}6 \mathrm{yr} \\
12 \mathrm{yr}\end{array} \\
\end{array}$ & $\begin{array}{l}7,000,000 \\
7,800,000\end{array}$ & $\begin{array}{l}70 \\
80\end{array}$ & & 8 & \\
\hline Postoperative & $4,400,000$ & 52 & 2 to 16 & 15 & 14 \\
\hline
\end{tabular}

(2) Right atrial angiocardiography showed that, except for a slight flow along the catheter (Figs 10 and 11), the contrast modium did not flow back from the right atrium to the inferior vena cava because the valve homograft performed its antireflux function perfectly. The valvular sinus is well seen on the angiocardiogram (lateral view). The contrast medium flowed through the left pulmonary artery and passage though the left lung was unimpeded.

CASE 2 Our second patient, J.B., was a 36-year-old woman, who had been cyanotic since she was a child but was normally developed.

At the age of 18 years she had had a cerebral abscess which was drained without sequelae.

At the age of 30 years she was referred to hospital because of exertional dyspnoea. Marked generalized cyanosis and clubbing of the fingers were noted. A very loud systolic murmur was heard to the left of the sternum, but there were no signs of cardiac failure.

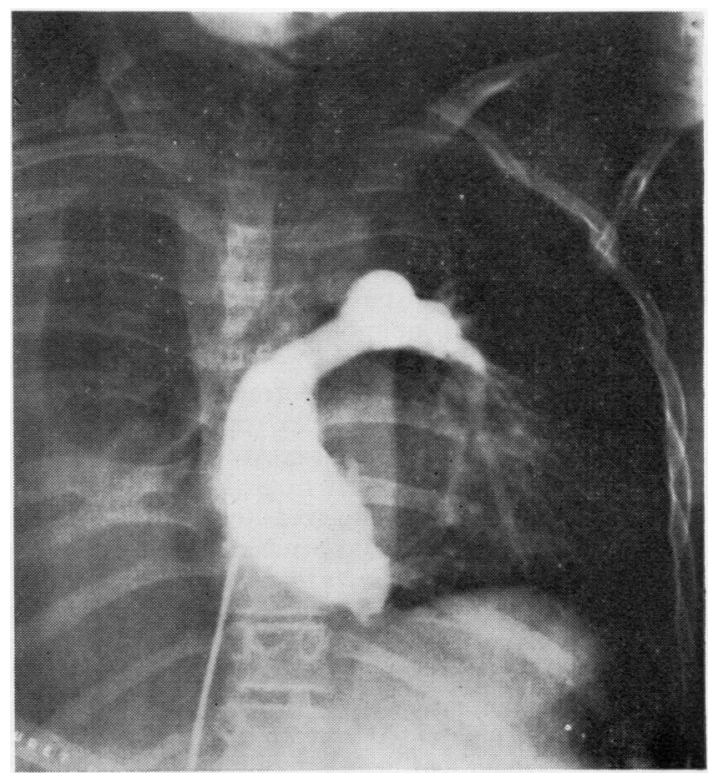

FIG. 10. Case 1. Postoperative right atrial angiocardiogram, anteroposterior view, showing opacification of right atrium and contrast medium flowing through left pulmonary artery.

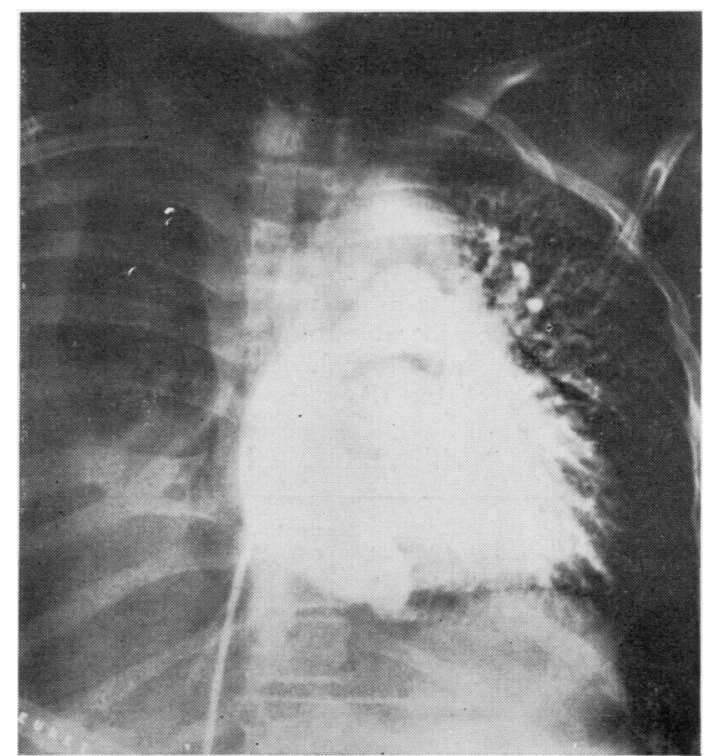

FIG. 11. Case 1. Postoperative right atrial angiocardiogram, anteroposterior view: passage of contrast medium through left lung is fast, as proved by good opacification of left atrium. Except for a slight flow along catheter, contrast medium does not flow back from right atrium to inferior vena cava, because the pulmonary valve homograft performs its antireflux function perfectly. 
At the age of 33 years exertional dyspnoea, nonproductive cough, and headache appeared, but they were improved by symptomatic therapy.

Finally, at the age of 36 years, her exercise tolerance was reduced and dyspnoea became progressively worse. She was admitted to hospital in January 1970 .

Cardiac catheterization and angiocardiography confirmed a type IIB tricuspid atresia, with pulmonary arteries which seemed of small size when compared with an enormous transposed aorta, but the right and left branches of the pulmonary artery were, in fact, of nearly normal size.

Operation (Fig. 1) was performed on 20 January, 1970. The pulmonary arterial pressure was 35.0 mmHg. After superior vena cava to right pulmonary artery anastomosis had been carried out (Fig. 2), the superior vena caval pressure was $10.0 \mathrm{mmHg}$. Anastomosis of the right atrial appendage to the proximal end of the right pulmonary artery was carried out by means of an aortic valve homograft (Fig. 3). Under cardiopulmonary bypass (Fig. 5) the atrial septal defect was closed, the pulmonary valve homograft was inserted into the inferior vena cava, and finally the main pulmonary artery was ligated. After the cardiopulmonary bypass had been discontinued, the superior vena cava was transected below the cava-pulmonary anastomosis (Fig. 6) at its entry into the right atrium.

The initial postoperative course was very satisfactory: cyanosis disappeared; the patient was in sinus rhythm at 80 per minute and ventilation and diuresis were excellent. To maintain an adequate blood pressure it was necessary to produce hypervolaemia by increasing the transfusion rate and tachycardia by isoproterenol.

A superior vena caval syndrome appeared eight days after the operation but disappeared after a few days under treatment with diuretics, while a bilateral serosanguineous pleural effusion required aspiration. On getting up, the patient had no oedema of the inferior limbs and only a moderate hepatomegaly. There was a considerable biological improvement: the blood count was $4,200,000 \mathrm{RBC} / \mathrm{mm}^{3}$ and haematocrit was $50 \%$ (Table II).

\section{T A B L E I I}

\begin{tabular}{|c|c|c|c|c|c|}
\hline & \multirow{2}{*}{$\underset{\text { per } \mathrm{mm}^{3}}{\mathbf{R B C}}$} & \multirow{2}{*}{$\begin{array}{c}\text { Haemato- } \\
\text { crit } \\
\%\end{array}$} & \multicolumn{3}{|c|}{ Pressure $(\mathrm{mmHg})$} \\
\hline & & & SVC & IVC & PA \\
\hline Preoperative $\mid \begin{array}{l}30 \mathrm{yr} \\
36 \mathrm{yr}\end{array}$ & $\begin{array}{l}6,750,000 \\
7,200,000\end{array}$ & $\begin{array}{l}70 \\
80\end{array}$ & 5 & & 35 \\
\hline Postoperative & $4,200,000$ & 50 & 10 & 15 & 30 \\
\hline
\end{tabular}

It is too soon yet to carry out cardiac catheterization, but these haemodynamic and angiocardiographic investigations will be performed shortly. The clinical course is quite satisfactory 10 months postoperatively: there is no cyanosis and no systolic murmur; cardiac auscultation is normal except for an accentuated second heart sound. There is no venous stasis in the upper half of the body, no pleural effusion, and no oedema of the inferior limbs. There is only a moderate persistent hepatomegaly.

CASE 3 The third patient, N.B., was a 23-year-old woman. Dyspnoea and cyanosis had appeared in childhood. An episode of cardiac failure occurred during pregnancy, in 1969 , followed by a premature birth four months before her admission to hospital in December 1969.

Physical examination showed clubbing of the fingers, cyanosis of the extremities, and hepatomegaly. A loud systolic murmur was heard in the whole precordial area. A blood count showed 3,700,000 RBC/ $\mathrm{mm}^{3}$ and the haematocrit was $44 \%$.

A diagnosis of tricuspid atresia with dextrocardia, atrial septal defect, and ventricular septal defect was made. There was a low pulmonary arterial pressure of $23.0 \mathrm{mmHg}$ (Table III).

T A B L E I I I

\begin{tabular}{|c|c|c|c|c|}
\hline & \multicolumn{4}{|c|}{ Pressure $(\mathrm{mmHg})$} \\
\hline & $\mathbf{R A}$ & & PA & \\
\hline \multirow[t]{2}{*}{$\begin{array}{c}\text { Preoperative } \\
\text { Maximal } \\
\text { Minimal } \\
\text { Mean }\end{array}$} & 7 & & $\begin{array}{l}23 \\
13 \\
16\end{array}$ & \\
\hline & SVC-RPA & IVC & $\mathbf{R A}$ & $\overrightarrow{\text { LPA }}$ \\
\hline $\begin{array}{l}\text { Postoperative } \\
\text { Maximal } \\
\text { Minimal } \\
\text { Mean }\end{array}$ & $\begin{array}{l}15 \\
10\end{array}$ & $17 \cdot 5$ & $\begin{array}{l}22.5 \\
17 \cdot 5\end{array}$ & $\begin{array}{l}17 \cdot 5 \\
12.5\end{array}$ \\
\hline
\end{tabular}

Operation was performed in March 1970. First, a superior vena cava to pulmonary artery anastomosis was carried out ; then, an anastomosis of the right $\underset{\times}{\sim}$ atrium to the proximal end of the right pulmonary $\frac{0}{\sigma}$ artery, using an aortic valve homograft ; finally, under 3 . cardiopulmonary bypass, a pulmonary valve homograft was inserted into the inferior vena cava, the atrial septal defect was closed, and the main pulmonary artery was transected and sutured.

The initial postoperative course was satisfactory, $\supset$ with good cardiac action. But, despite blood overcompensation and isoproterenol, her pulse and blood N pressure fell slowly 6 hours postoperatively and she 0 died.

At necropsy there was no thrombosis and the anas- N tomoses were patent; but the mitral valve was $\frac{\omega}{\sigma}$ abnormal, with vegetations and a perforation of 12 $\mathrm{cm}^{2}$ in the anterior leaflet. Finally, the right atrium was small and its wall was very thin.

We are of the opinion that failure was due to this $\stackrel{\oplus}{+}$ mitral insufficiency.

\section{DISCUSSION}

A new surgical technique for repair of tricuspid $\stackrel{\mathbb{D}}{\varrho}$ atresia seems worth while. Indeed, in reviewing the literature on the subject, no corrective tech-o 
nique is mentioned, only palliative procedures.

Some years ago, after the appearance of the first papers on cava-pulmonary anastomosis (Glenn and Patiño, 1954; Glenn, 1958), we conceived the theoretical basis of the operation we report. Experimental research on dogs enabled us to check the technical feasibility of this procedure, but there were no survivals for more than a few hours, perhaps because the haemodynamic status of a normal dog heart does not allow a circulation which involves circulatory bypass of the right side of the heart. We were of the opinion that the right atrium of a normal heart could not provide the required work, whereas a hypertrophied right atrium, as in tricuspid atresia, could supply the additional work represented by a pulmonary arterial pressure higher than the left atrial pressure. However, it seemed to us indispensable to provide the right atrium with valve homografts, one inserted into the inferior vena cava at the level of the right atrium, and the other at the exit from the right atrium to the left lung, to prevent free flow between the inferior vena cava, the right atrium, and the pulmonary artery and, in this way, stasis in the lower half of the body and inadequate cardiac filling.

The pliability and plasticity of the homograft facilitates suturing; but the long-term fate of valve homografts is unknown. Rastelli, Wallace, and Ongley (1969) have reported that calcification of the aortic wall of homografts used to repair truncus arteriosus defects has occurred in each of five patients operated on, without preventing continued function of the leaflets.

Reports from other surgeons (Bigelow et al., 1967 ; Bigelow, 1968) on the fate of aortic homografts, in place for as long as 13 years in the thoracic aorta, have indicated that the segment of aorta and aortic valves have remained functionally satisfactory with time. Indeed, secondary calcifications seem to occur only in aortic valve homografts; pulmonary valve homografts are rarely affected by these changes and they can be used not only for 'valvation' of the inferior vena cava, but also for an anastomosis between the right atrium and the proximal end of the right pulmonary artery. Barratt-Boyes et al. (1969) and Ross (1971) have noted that fresh aortic valve homografts are less likely to become calcified than those that are sterilized and preserved.

A homograft between the right atrium and the proximal end of the right pulmonary artery is not indispensable, because in our first patient we did not use one for want of having one small enough. The result was satisfactory two and a half years postoperatively. But we are of the opinion that the homograft is probably useful, for the postoperative course was more difficult in this patient and an inferior vena caval syndrome was observed (hepatomegaly, melaena, oligoanuria), though this last syndrome could be explained by the child's congenital bilateral hydronephrosis.

A striking fact in the postoperative course was the need to provide a large amount of fluid infusion (blood and physiological solution) and maintain a tachycardia to ensure a correct haemodynamic balance. During the first three days postoperatively we had to ensure an overcompensation and a tachycardia (about 100 to $120 / \mathrm{min}$ ) as if the right atrium, 'ventricle-like', could supply a satisfactory left pulmonary blood flow only by ensuring a venous hyperpressure which would help filling and a tachycardia permitting a suitable flow, until a spontaneous balance was obtained. The need for a large volume of fluid infusion is well known in cava-pulmonary anastomosis and is explained by a liquid storage in the upper half of the body. This new technique, which is a double cava-pulmonary anastomosis, could only aggravate this syndrome.

Respiratory assistance should be stopped early because positive pressure prevents central venous return.

Another less explicable feature of the postoperative course was, in both cases, a right or bilateral pleural effusion which required a few pleurocenteses.

The operation is not technically difficult. We have waited for as long as 30 months after operation before reporting this technique. The immediate result is remarkable and remains satisfactory. One element remains unpredictable - the haemodynamic consequences of an eventual atrial rhythm disturbance such as an atrial fibrillation or flutter.

\section{INDICATIONS}

The indications for this corrective procedure, though remaining limited, apply to many patients. Our first two patients were anatomically and haemodynamically privileged; they had pulmonary arteries of normal size and low pressure.

The anatomical classification of tricuspid atresia, from Edwards and Burchell (1949) (Fig. 12) and Keith, Rowe, and Vlad (1958), distinguishes two principal types-type I, with normally related great arteries, and type II, with transposition of the great arteries ; and three groups in each typegroup A, with pulmonary atresia, group B, with 


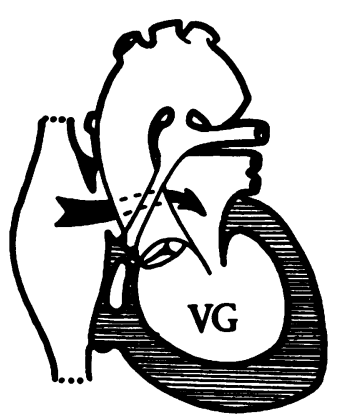

IA.

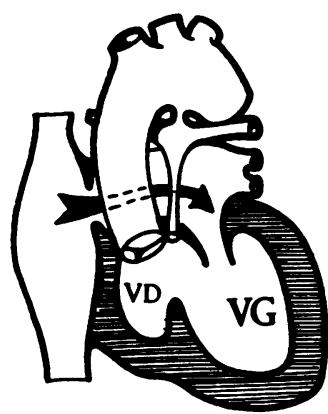

II A.

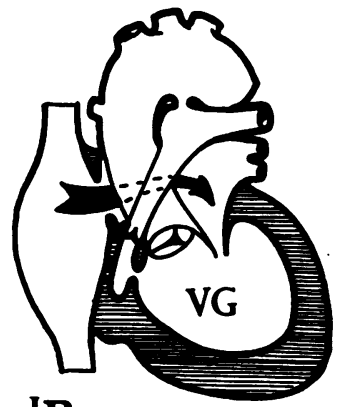

IB.

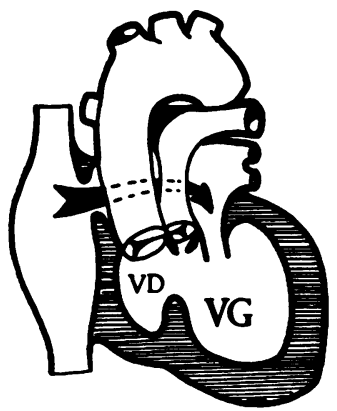

IIB.

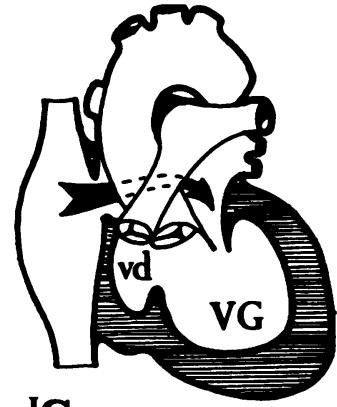

IC.

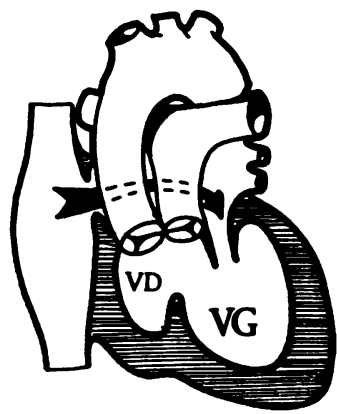

IIC.

FIG. 12. Drawing illustrates the different types of tricuspid atresia (from Edwards and Burchell, 1949).

pulmonary valvular or subvalvular stenosis, and group $\mathrm{C}$, with a normal pulmonary artery and increased pulmonary blood flow.

Most of the children with tricuspid atresia have a poor prognosis and die rather early. In these circumstances, only palliative surgical procedures can be considered (anastomosis in groups $\mathrm{A}$ and $B$ and banding of the main pulmonary artery in group C), but we are of the opinion that they could profit from this corrective procedure as soon as they are older and have a bodily development compatible with the anatomical, haemodynamic, and technical necessities of this operation.

\section{REFERENCES}

Barratt-Boyes, B. G., Roche, A. H. G., Brandt, P. W. T., Smith, J. C., and Lowe, J. B. (1969). Aortic homograft valve replacement. A long-term follow-up of an initial series of 101 patients. Circulation, 40, 763.

Bigelow, W. G. (1968). Personal communication.
Trimble, A. S., Aldridge, H. E., Bedard, P., Spratt, E. H., and Lansdown, E. L. (1967). The problem of insufficiency following homograft replacement of the aortic valve. J. thorac. cardiovasc. Surg., 54, 478.

Edwards, J. E., and Burchell, H. B. (1949). Congenital tricuspid atresia: a classification. Med. Clin. N. Amer., 33, 1177.

Glenn, W. W. L. (1958). Circulatory bypass of the right side of the heart. IV. Shunt between superior vena cava and distal right pulmonary artery-Report of clinical application. New Engl. J. Med., 259, 117.

- and Patiño, J. F. (1954). Circulatory by-pass of the right heart. Yale J. Biol. Med., 27, 147.

Keith, J. D., Rowe, R. D., and Vlad, P. (1958). Heart Disease in Infancy and Childhood. Macmillan, NewYork.

Rastelli, G. C., Wallace, R. B., and Ongley, P. A. (1969). Complete repair of transposition of the great arteries with pulmonary stenosis. A review and report of a case corrected by using a new surgical technique. Circulation, 39, 83.

Ross, D. N. (1971). Aortic valvar replacements. In Proceedings VI World Congress of Cardiology, London, September 1970. Brit. Heart J., 33 (Suppl.), 39. 